\title{
Algorithms for Structural and Dynamical Polychronous Groups Detection
}

\author{
Régis Martinez ${ }^{1}$ and Hélène Paugam-Moisy ${ }^{2}$ \\ ${ }^{1}$ LIRIS, UMR CNRS 5205, Université de Lyon, F-69676 Bron, France \\ ${ }^{2}$ TAO - INRIA, LRI - Université Paris-Sud 11, F-91405 Orsay, France \\ regis.martinez@liris.cnrs.fr, hpaugam@lri.fr
}

\begin{abstract}
Polychronization has been proposed as a possible way to investigate the notion of cell assemblies and to understand their role as memory supports for information coding. In a spiking neuron network, polychronous groups (PGs) are small subsets of neurons that can be activated in a chain reaction according to a specific time-locked pattern. PGs can be detected in a neural network with known connection delays and visualized on a spike raster plot. In this paper, we specify the definition of PGs, making a distinction between structural and dynamical polychronous groups. We propose two algortihms to scan for structural PGs supported by a given network topology, one based on the distribution of connection delays and the other taking into account the synaptic weight values. At last, we propose a third algorithm to scan for the PGs that are actually activated in the network dynamics during a given time window.
\end{abstract}

\section{Introduction}

One of the main challenges in cognitive science is to understand how knowledge is represented and processed in the brain. From the early notions of cell assemblies [5] and "grand-mother cells" (see [4]), many open questions are still debated. What is the support of memory? How and where information is coded in the brain activity? The recent hypothesis that information could be encoded by precise spike timings gives arguments for the thesis of temporal rather than spatial cell assemblies. Pointing out the fact that connection delays have non uniform values between neurons in the brain, Izhikevich proposed the concept of polychronization [7], which is far richer than the current concepts of synchronization and synfire chains [1]. Also in computer science the concept of polychronization yields valuable tracks for defining new learning rules in spiking neuron networks [10] and polychronous groups have been confirmed to play the role of dynamical cell assemblies in a classification task [9]. Studying PGs and understanding their role in information coding could help improving both the network structure and the effectiveness of learning rules acting on delays.

So far, no formal definition has been given for a polychronous group, and only specific methods to inventory them have been proposed [6]8]. In section 2]we give a precise definition of a polychronous group (PG), making a distinction between structural and dynamical PGs. In section 3 we present three algorithms and data structures to inventory all polychronous groups of a given network topology and to detect which groups

C. Alippi et al. (Eds.): ICANN 2009, Part II, LNCS 5769, pp. 75-84, 2009.

(C) Springer-Verlag Berlin Heidelberg 2009 
are triggered in spike activity. In section 4 we give a complexity analysis of the algorithms, and we present experimental measurements: Number of PGs (mean values, from several experiments) when varying different parameters.

\section{Definition of Polychronous Groups}

\subsection{PG Definition}

In the founding paper [7] polychronization denotes the fact that several neurons can be activated in a chain reaction according to a specific time-locked pattern of firings, not only in pure synchrony. A polychronous group is characterized by a precise temporal pattern of firings, in a subset of neurons, that is more likely to happen than just by chance in neural activity. Such patterns are intrinsically based on the network topology: connectivity, synaptic weights and especially conduction delays.

In recent work [8] a polychronous group, referred to as a polygroup 1 , is defined as the set of neurons that supports the time-locked pattern. We state that the set of neurons involved in the temporal pattern is not enough to characterize the PG. Indeed, if one neuron can appear in more than one PG, it is clear that a given set of neurons could also fire with several different timings, and support or participate to more than one PG. The chain reaction that defines a PG is a series of causal interactions between neurons, such as $\left[N_{1}, t_{1}\right],\left[N_{2}, t_{2}\right], \ldots,\left[N_{i}, t_{i}\right] \Rightarrow\left[N_{j}, t_{j}\right]$, where a pair $\left[N_{k}, t_{k}\right]$ denotes a spike fired by neuron $N_{k}$ at time $t_{k}$. The chain is started by a specific firing pattern of a small number $s$ of neurons, the triggering neurons, further named triggers. Hence we propose to define a PG by a list of triggers associated to a temporal firing pattern:
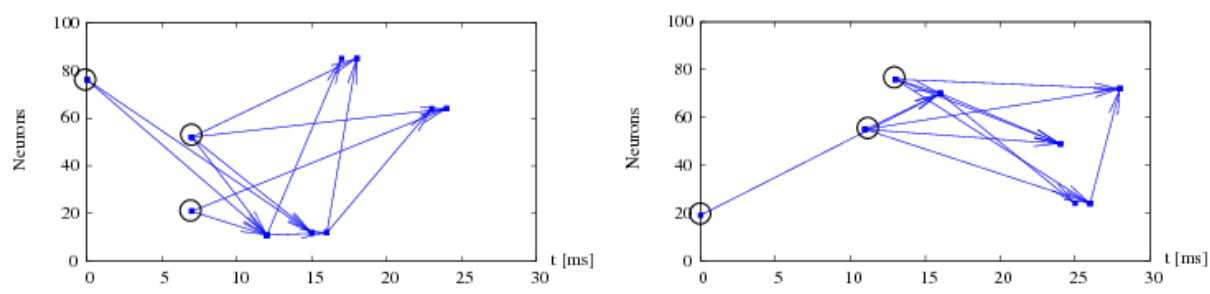

Fig. 1. Graphs for two polychronous groups: the $21-52-76(7,7,0)$ (left PG) and the $19-55-76(0,11,13)$ (right PG). They share neuron 76 among their respective sets of triggers.

Definition 1. A s-triggered polychronous group refers to the set of neurons that can be activated by a chain reaction whenever the triggers $N_{k}(1 \leq k \leq s)$ fire according to the timing pattern $t_{k}(1 \leq k \leq s)$. The $P G$ is denoted by: $\quad N_{1}-N_{2}-\ldots-N_{s}\left(t_{1}, \ldots, t_{s}\right)$ where the firing times $t_{k}$ are listed in the same order as the corresponding triggers $N_{k}$.

${ }^{1}$ We do not use the term polygroup because it might bring confusion with other uses in Physics. 
To decide whether a neuron could be activated by counting the number of spikes it recieves simultaneoulsy, we follow Izhikevich [7] by extending the notion of simultaneous arrival to a short time range denoted $j$ itter.

A graphical representation of a PG can be plotted on a spike raster plot of the network activity, focusing attention on a subset of neurons, and drawing additional links for representing the causality of interactions (see Figure 1). Such a representation is a subgraph of the neural activity, where a vertex is a pair $\left[N_{k}, t_{k}\right]$, and a directed edge denotes the causal influence from a pre-synaptic neuron to a post-synaptic one.

\subsection{Structural PGs vs. Dynamical PGs}

Definition 1 allows to inventory all the possible $s$-triggered polychronous groups supported by a given spiking neuron network with known connectivity and conduction delays, disregarding weight values. Since such PGs depend on the network architecture only, they are structural and we call them the supported PGs.

However, synaptic weights are usually subject to learning rules and their values change through time, which can influence the decision whether a certain amount of spikes simultaneoulsy incoming to a neuron $N_{j}$ is sufficient or not for triggering a spike fired by $N_{j}$. Then we define adapted PGs by applying Definition 1 in taking into account the membrane potential dynamics of neurons and the values of synaptic weights for finding the causal relations yielding a neuron $N_{j}$ to actually fire a spike at time $t_{j}$. Since activated PGs do not depend on the network dynamics (e.g. under the influence of a given input), they are also structural PGs.

Another question is to find which PGs actually appear in the spike activity of a neuron network during a given time window. These PGs are a subset of the structural PGs and we call them activated PGs. Unlike structural PGs, activated polychronous groups are dynamical PGs, since they depend on the network dynamics.

\section{Scanning for Polychronous Groups}

Algorithms 1 and 2 are designed to scan for supported PGs and adapted PGs respectively. They are based on a given network topology, with known connectivity, conduction delays and synaptic weights. In Algorithm 1, all the combinations of $s$ neurons are tested as possible triggers, under the hypothesis that NbSpikesNeeded are required to generate a causal relation making a neuron $N_{j}$ to spike ( $s$ and NbSpikesNeeded are set by the user). Actual post-synaptic potentials (PSPs) are not taken into account in Algorithm 1 whereas in Algorithm 2, the decision to let a neuron $N_{j}$ spike requires the computation of the weigthed PSPs recieved by $N_{j}$. In Algorithm 2, the amount of incoming spikes can differ from NbSpikesNeeded, a parameter which is no longer useful ( $s$ is still required and set by the user). Algorithm 3 is written to scan for the actual appearance of previously inventoried adapted polychronous groups in the network activity during a time slice of simulation data (with varying inputs, for instance).

All three algorithms are suited for any model of neuron that can be run in eventdriven mode. Algorithm 2 uses the neuron model equation to decide whether the neuron spikes or not, on the basis of its recent PSP history. This constraint may be relaxed for 
Algorithm 1 that can be straightforward adapted to more general neuron models, since it is only based on spike events, and do not take care of the intrinsic dynamics of neurons.

The model neuron we used for experiments has the following characteristics (based on $S R M_{0}$ [2]): The firing threshold $\vartheta$ is set to a fixed negative value (e.g. $\vartheta=-50 \mathrm{mV}$ ) and the threshold kernel simulates an absolute refractory period $\tau_{a b s}$, when the neuron cannot fire again, followed by a reset to the resting potential $u_{\text {rest }}$, lower than $\vartheta$ (e.g. $u_{\text {rest }}=-65 \mathrm{mV}$ ). We assume that the simulation is computed in discrete time (with $0.1 \mathrm{~ms}$ time steps). The variables of each neuron are updated at each new incoming spike (event-driven programming), which is sufficient for computational purpose.

The network structure is typical of Reservoir Computing methods: Connections between neurons are drawn randomly, according to a given connectivity; Weights start from 0.5 as initial value and can vary under STDP, a temporal Hebbian rule of synaptic plasticity; Delays are fixed but random, between 1 and $20 \mathrm{~ms}$.

\subsection{Definitions}

\section{Notations}

$n:$ number of neurons in the network

$N_{i}$ : neuron numbered $i$

$N_{T_{k}}$ : triggering neuron numbered $T_{k}$, with $k$ from 1 to $s$

$t:$ current time (virtual biological time, in simulations)

$d_{i j}$ : conduction delay on the connection from $N_{i}$ to $N_{j}$

$w_{i j}$ : synaptic weight from $N_{i}$ to $N_{j}$, equals 0 if connection does not exist.

\section{Data structures}

Post-Synaptic Potentials. A PSP is denoted $P S P_{t_{p s p}, N_{l}, N_{m}}$ : Post-Synaptic Potential evoked at time $t_{p s p}$ by a pre-synaptic neuron $N_{l}$ on a post-synaptic neuron $N_{m}$.

Event queue. The queue EventQueue is the structure for processing simulation events (evoked PSPs). It contains the events ordered by their chronological occurence in the future.

PSP list stored in neurons. For the purpose of our algorithms, we need to store, for each neuron, the list of the spikes it recieved during a given time course elapsed, the Jitter (see Section 2.1). This list is called PSPList ${ }_{i}$, for neuron $N_{i}$.

Data structure for a PG. When a PG is calculated, informations have to be stored :

- timings $t_{T_{1}}, \ldots, t_{T_{s}}$ of the triggers $N_{T_{1}}$ to $N_{T_{s}}$;

- for each PSP, $P S P_{t_{p s p}, N_{l}, N_{m}}$, the ID of $N_{m}$ and its spike-firing time, the ID of $N_{l}$ and the time of evocation of the PSP.

\section{Variables}

NbTriggeringConnections $s_{i}$ : number of connections received by $N_{i}$ from triggers

MaxPotential $_{i}$ : maximum membrane potential that neuron $N_{i}$ might reach under the action of triggers

tLastSpike L $_{i}$ : time of most recent firing of neuron $N_{i}$, initialized to - RefractoryPeriode, so that previous spikes are already out of refractory periode 


\section{Parameters}

$s:$ number of triggers, fixed for every polychronous groups

NbSpikesNeeded : number of simultaneous impinging spikes necessary to trigger a new spike in any neuron

Jitter : time range for spikes to be considered as "simultaneous"

NbSpikesMax : maximum number of spikes in a polychronous group

NbSpikeMin : minimum numer of spikes in a polychronous group for it to be saved

MaxTimeSpan : maximum time span of the polychronous group

MinTimeSpan : minimun time span of the polychronous group

PSPStrength : amplitude of a Post Synaptic Potential (PSP)

RestingPotential : default membrane potential of a neuron when it has no input

Threshold: membrane potential value above which the neuron spikes

RefractoryPeriode : time after a spike, during which a neuron cannot fire again

\subsection{Algorithms}

Algorithm 1. In order to list the supported PGs, we first look at all the combinations of a given number $s$ of neurons. We check each combination, looking for neurons that might be excited enough to fire in turn, because they recieve more than a certain amount of spikes, NbSpikesNeeded. If such neurons exist, then the combination becomes a set of triggers. We simulate the firing of the triggers with the right starting timing and record the propagation of the neural activity, until it dies or it reaches an upper limit MaxTimeSpan set for the time span of a PG. Moreover, we limit the record to a given number of neurons NbSpikesMax in order to truncate the possible cyclic PGs.

In this algorithm, the propagation of the activity is based on the number of spikes recieved by each neuron in a time window. For instance, the neurons may be parameterized so that they fire whenever they are impinged by at least three spikes within a millisecond. A full description, in pseudo-language, is given in Annex.

Algorithm 2. The principle of this algorithm is very similar to the previous one, except that the decision of firing or not is based on the level of the membrane potential, which depends on (a) the weights of the incoming connections that will modulate the increase of the membrane potential and thus the probability to generate a new spike and (b) the elapsed time since the previous PSP.

As in Algorithm 1, we look at all the possible combinations of $s$ triggers, except that neuron activity is calculated upon its membrane potential exceeding or not the firing threshold. In this algorithm, the propagation of the activity is based on the fact that the mambrane potential exceedes the threshold. See Annex.

Algorithm 3. Algorithm 3 is written to scan for the appearance of known polychronous groups (already detected by Algorithm 1 or 2) in the activity recorded from a simulation, during a given time range.

In order to detect the activation of a PG in a particular time window in the recorded activity of a simulation (Algorithm 3), it would be ideal to check if the whole group is activated. For sake of computational time, we only look for the firing of the triggers of 
the PG, with the good timing pattern within a precision of Jitter. We based this algorithmic simplification on the assumption that the activation of the triggers will activate the tail of the polychronous group with little change, which is likely if the known PG has been previously detected to be an adapted PG, but could fail in case of supported PGs.

1: // We look for the actual activation of known PGs in a temporal range [Start; End]. For each neuron $N_{i}$, the list of spikes fired by this neuron between times Start and End is stored already (i.e. we assume that the spike raster has been recorded).

2: Let Spike $_{m i}$ be the $m^{\text {th }}$ spike of the list of spikes fired by $N_{i}$, at time $t_{\text {Spike }_{m i}}$

3: for all PG triggered by $\left\{N_{T_{1}}, N_{T_{2}}, \ldots, N_{T_{s}}\right\}$ with timing $\left\{t_{T_{1}}, t_{T_{2}}, \ldots, t_{T_{s}}\right\}$ do

4: $\quad \forall$ Spike $_{m T_{1}}$ at time $t_{\text {Spike }_{m T_{1}}}$

5: $\quad$ if $\forall k \in\left[T_{2} ; T_{s}\right], \exists S p i k e_{m T_{k}}$ at time $t_{\text {Spike }_{m T_{1}}}+\left(t_{T_{k}}-t_{T_{1}}\right)$ then

6: $\quad$ Save activation of PGs at time $t_{\text {Spike }_{m T_{1}}}-t_{T_{1}}$

7: $\quad$ end if

8: end for

\section{Complexity Study and Experiments}

\subsection{Computational Complexity}

First, the complexity estimation for Algorithm 1 is developed below. The complexity of Algorithm 2 and Algorithm 3 will be discussed afterwards.

Let $c$ be the connectivity of a network, i.e. the probability that one neuron projects a connection to another. Let $A C=(n-1) \times c$ be the average number of connections recieved by any neuron in the network. Remind that $s$ is the number of triggers.

The number of combinations to parse is $C_{n}^{s}=\frac{n !}{s !(n-s) !}$. For each combination, we search for neurons that recieve concurrent connections from triggers and count such connections. This step is computed with complexity $O(n+s \times A C)$.

The probability that a neuron recieves $k=N b$ Spikes Needed connections from other neurons, with $k \leq s$, is $c^{k}$. We watch which neurons have enough input connections to trigger a new spike. For each excited neuron, we initialise the calculation of the corresponding $\mathrm{PG}$ and enqueue the spike events, i.e. $s \times A C$ operations.

The while block is the most difficult to evaluate because of the various parameters it envolves. In worst case, all $n$ neurons will recieve $N b S p i k e s N e e d e d$ spikes, and spike in turn. Then there would be $n \times k$ events to process, and $n \times A C$ new PSP to enqueue. Neurons would spike again as soon as they can, in regard to their refractory period. There should be at most $M / R \times n \times A C$ events to process in the whole calculation, where $M=$ MaxTimeSpan and $R=$ RefractoryPeriode.

Hence, the overall complexity $X_{\text {algo } 1}$ of Algorithm 1, in worst case, is :

$$
\underbrace{C_{n}^{s}}_{\text {combin. }} \times[\underbrace{(n+s \times A C)}_{\text {search triggers }}+\underbrace{(s \times A C) \times\left(c^{k}\right)}_{n b \text { triggers }} \times(\underbrace{s \times A C}_{\text {init. spikes }}+\underbrace{M / R \times n \times A C)}_{\text {worst case events }}]
$$


Since $s \ll n$, the number of combinations $C_{n}^{s}$ can be approximated by $\left(\frac{n}{s}\right)^{s}$ and $A C=(n-1) \times c$ replaced by $c \times n$, which yields an upper bound for $X_{a l g o 1}$ :

$$
X_{\text {algo } 1} \leq \frac{1+s \times c}{s^{s}} n^{s+1}+\frac{c^{k+2}}{s^{s-2}} n^{s+2}+\frac{M}{R} \times \frac{c^{k+2}}{s^{s-1}} n^{s+3}
$$

It results that the complexity $X_{\text {algo } 1}$ is of order $O\left(n^{s+3}\right)$ in worst case. In practical cases, spiking neuron networks usually have a sparse connectivity. Fixing the order of magnitude of the connectivity $c$ to $1 / \sqrt{n}$ looks like a realistic estimation, both for artificial networks (e.g. $c=0.1$ in a network of 100 neurons, when computing with spiking neuron networks) and biological networks (around $10^{5}$ connections per neuron between $10^{11}$ neurons in the human brain). Replacing $c$ by $n^{-1 / 2}$ in Equation (1) gives:

$$
X_{\text {algo } 1} \leq \frac{1}{s^{s}} n^{s+1}+\frac{1}{s^{s-1}} n^{s+1 / 2}+\frac{1}{s^{s+2}} n^{s}+\frac{M}{R} \times \frac{1}{s^{s-1}} n^{s+1} \text { since } k \geq 2
$$

Finally, in practical cases, the complexity of Algorithm 1 is of order $O\left(n^{s+1}\right)$.

The complexity $X_{\text {algo } 2}$ of Algorithm 2 is similar to $X_{\text {algo } 1}$ because both algorithms have the same control structure. Running Algorithm 2 should be slightly more time consuming if the neuron model is complex.

The complexity $X_{a l g o 3}$ of Algorithm 3 is of order $O(P * S / n)$, where $P$ is the number of known polychronous groups (computed by Algorithm 1 or 2), and $S$ the total number of spikes in the time slice chosen for scanning the network activity.

\subsection{Experiments}

A direct comparison with other algorithms is not straightforward since the Izhikevich's code available on [6] starts from cutting off the weights under an arbitrary value of 0.95 , and does not exactly compute any of the PG categories we defined. The Maier \& Miller's method [8] (noted "MM algo" in Table 1) is close to Izhikevich's one: both are based on a $n \times t_{\max }$ matrix of spike arrival counts, with the risk of consuming a huge amount of memory, since $t_{\max }$ is the maximum time to which the simulation is run.

In Table 1, NbSpikesNeeded $=s=3$. The first two lines show the strong influence of the connectivity $c$ on the number of PGs. In the next three lines, the network size is varied with an adapted value of $c$ in order to keep fixed the degree of the connection graph. Different parameters of the neuron model (with coherent value of Jitter) highly

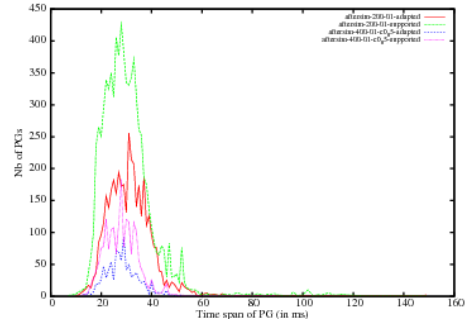

Fig. 2. PGs timespan comparison
Table 1. Experimental measurements

\begin{tabular}{|c|c|c|c|c|c|}
\hline$n$ & $c$ & Jitter & supported PGs & adapted PGs & MM algo. \\
\hline 100 & 0.1 & $1 \mathrm{~ms}$ & 13.6 & 13.2 & 13.6 \\
100 & 0.2 & $1 \mathrm{~ms}$ & 1295 & 1308 & 1300 \\
\hline 100 & 0.18 & $1 \mathrm{~ms} / 0.4$ & $697 / 697$ & $702 / 72$ & 732 \\
200 & 0.09 & $1 \mathrm{~ms} / 0.4$ & $295 / 295$ & $274 / 16$ & 289 \\
500 & 0.036 & $1 \mathrm{~ms} / 0.4$ & $103 / 103$ & $112 / 6$ & 107 \\
\hline 200 & 0.09 & $1.2 \mathrm{~ms}$ & 431 & & 434 \\
200 & 0.09 & $1.0 \mathrm{~ms}$ & 295 & & 289 \\
200 & 0.09 & $0.7 \mathrm{~ms}$ & 176 & & 167 \\
200 & 0.09 & $0.5 \mathrm{~ms}$ & 79 & & 67 \\
200 & 0.09 & $0.1 \mathrm{~ms}$ & 0 & & 0 \\
\hline
\end{tabular}


influence the number of adapted PGs (but not sructured PGs). The last line shows that an absence of tolerance ( Jitter $=0.1 \mathrm{~ms}=$ simulation time step) could lead to an absence of PGs. With NbSpikesNeeded $=2, n=100$ and $c=0.18$ (not in Table 1), there are 387 supported PGs with $s=3$ triggers, but only 4 with $s=2$.

Figure 2 shows that the distribution of the PGs timespans (time from the spike of the earliest trigger to the spike of the latest neuron belonging to the PG) is similar for supported and adapted PGs, for different network sizes. Moreover, such a time span distribution is comparable to Izhikevich's observation ([7], p.127).

\section{Discussion}

We have proposed to clarify the definition of a polychronous group and set a standard notation, taking into account both the set of triggers and their specific firing pattern. We make a distinction between three categories of PGs, whether they are scanned from the network architecture only, or they take into account the variations of weights under a learning algorithm, or they are scanned for reflecting the dynamical activity inside the network in response to input data. Though designed for the analysis of simulations with spiking neuron network models, the algorithms could also be applied to real data since multi-neuron activities appear to be recordable in natural neuron networks [3].

\section{References}

1. Abeles, M.: Corticonics: Neural Circuits of the Cerebral Cortex. Cambridge Press, New York (1991)

2. Gerstner, W., Kistler, W.: Spiking Neuron Models: Single Neurons, Populations, Plasticity. Cambridge University Press, Cambridge (2002)

3. Gourévitch, B., Eggermont, J.: Maximum decoding abilities of temporal patterns and synchronized firings. In: NeuroComp. 2008 (2008) hal-00331583

4. Gross, C.G.: Genealogy of the "grandmother cell". The Neuroscientist (2002)

5. Hebb, D.O.: The Organization of Behaviour. Wiley, New York (1949)

6. Izhikevich, E.M.:

http://vesicle.nsi.edu/users/izhikevich/ publications/spnet.htm (2006)

7. Izhikevich, E.M.: Polychronization: Computation with spikes. Neural Computation 18(2), 245-282 (2006)

8. Maier, W.L., Miller, B.N.: A minimal model for the study of polychronous groups. arXiv:0806.1070v1 [cond-mat.dis-nn] (2008); (presented at the TS4CF08 Meeting of The American Physical Society)

9. Martinez, R., Paugam-Moisy, H.: Les groupes polychrones pour capturer l'aspect spatiotemporel de la mémorisation. In: NeuroComp. 2008 (2008) hal-00331613

10. Paugam-Moisy, H., Martinez, R., Bengio, S.: Delay learning and polychronization for reservoir computing. Neurocomputing 71(7-9), 1143-1158 (2008)

\section{A Algorithm 1}

1: // Lines starting with // are comments.

2: for all combination of $s$ neurons out of $n$ neurons of the network do

3: // Look for PGs triggered by this combination 


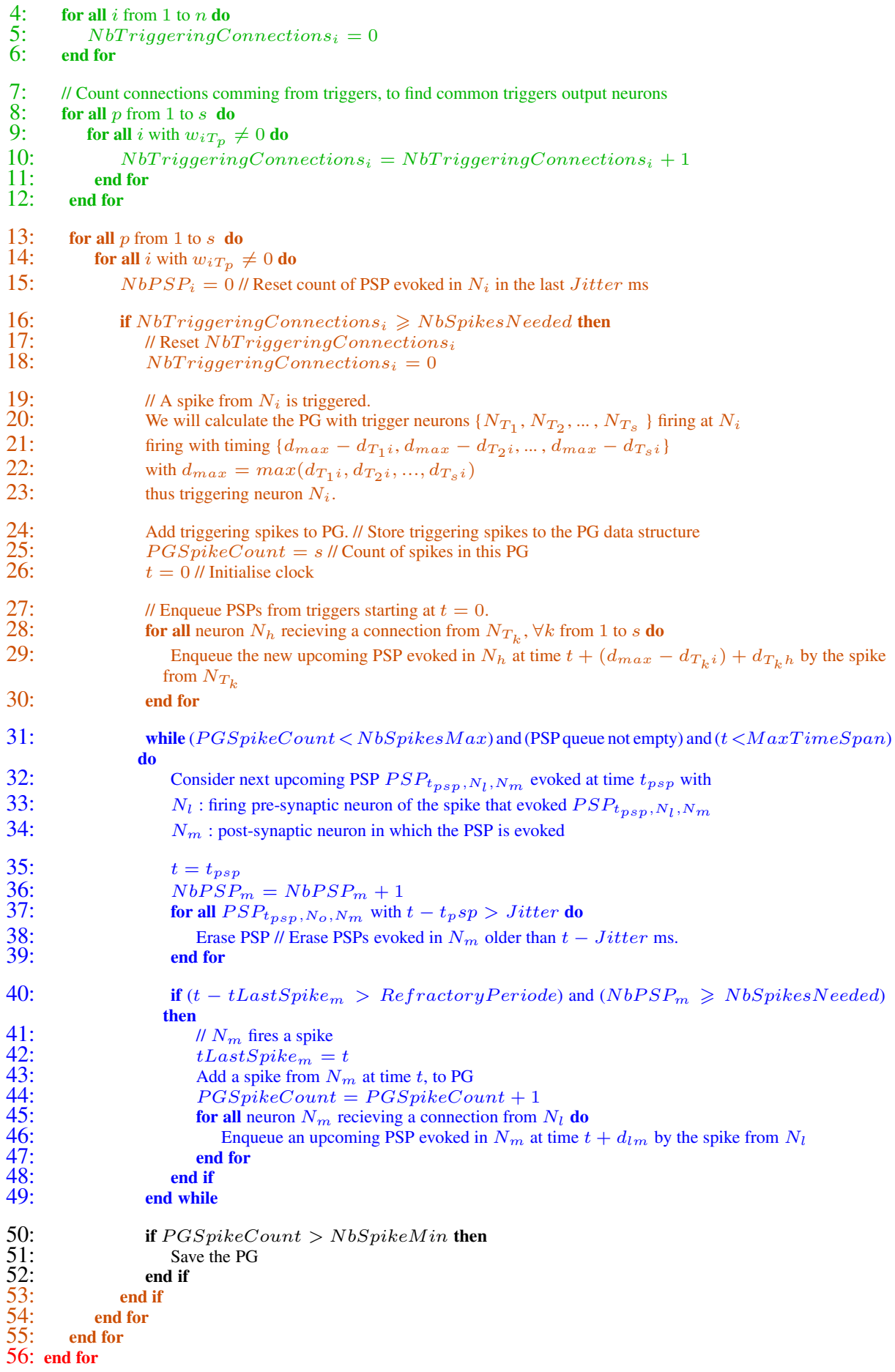




\section{B Algorithm 2}

\section{N.B. Red printing is for the lines that differ from Algorithm 1.}

1: for all combination of $s$ neurons out of $n$ neurons of the network do

2: // Look for PGs triggered by this combination

3: for all Neuron $N_{i}$, output of a triggering neuron do

4: $\quad \operatorname{NbPSP}_{i}=0 / /$ Count of PSP evoked in $N_{i}$ in the last Jitter ms

5: $\quad$ Potential $_{i}=$ RestingPotential // Set initial membrane potential for $N_{i}$

6: $\quad$ MaxPotential $_{i}=$ Potential $_{i}+\sum_{T_{j}} w_{T_{j}}$ PSPStrength

7: $\quad$ if MaxPotential $_{i} \geqslant$ Threshold then

8: $\quad$ // A spike from $N_{i}$ is triggered.

9: We will calculate PG with trigger neurons $\left\{N_{T_{1}}, N_{T_{2}}, \ldots, N_{T_{s}}\right\}$ firing at $N_{i}$

10: $\quad$ firing with timing $\left\{d_{\max }-d_{T_{1} i}, d_{\max }-d_{T_{2} i}, \ldots, d_{\max }-d_{T_{s} i}\right\}$

11: $\quad$ with $d_{\max }=\max \left(d_{T_{1} i}, d_{T_{2} i}, \ldots, d_{T_{s} i}\right)$

12: $\quad$ thus triggering neuron $N_{i}$.

13: $\quad$ Add triggering spikes to PG. // Store triggering spikes to PG data structure

14:

15: PGSpikeCount $=0 / /$ Count of spikes in this PG $t=0$

16:

17:

// Enqueue PSPs from triggers

for all neuron $N_{h}$ recieving a connection from $N_{T_{h}}$

19:

Enqueue the new upcoming PSP evoked in $N_{h}$ at time $t+\left(d_{\text {max }}-d_{T_{k} i}\right)+d_{T_{k} h}$ by the spike from $N_{T_{k}}$ end for

20:

while (PGSpikeCount $<$ NbSpikesMax) and (PSP queue not empty) and ( $t<$ MaxTimeSpan) do

Consider next upcoming PSP $P S P_{t_{p s p}, N_{l}, N_{m}}$ evoked at time $t_{p s p}$ with

22 .

$N_{l}$ : firing pre-synaptic neuron of the spike that evoked $P S P_{t p s p}, N_{l}, N_{m}$

$N_{m}$ : post-synaptic neuron in which the PSP is evoked

$t=t_{p s p}$

25:

for all $P S P_{t_{p s p}, N_{o}, N_{m}}$ with $t-t_{p} s p>J i t t e r$ do

26:

27:

Erase PSP // Erase PSPs evoked in $N_{m}$ older than $t-$ Jitter ms. end for

28:

29:

// Re-evaluate decreasing membrane potential, with regard to last spike impact recieved $t^{f}$

Potential $_{m}=\eta\left(\right.$ Potential $\left._{l}, t^{f}\right)$

Potential $_{m}=$ Potential $_{m}+w_{l m} \times$ PSPStrength

31:

32:

if Potential $_{m} \geqslant$ Threshold $)$ then

// $N_{m}$ fires a spike

Add a spike to from $N_{m}$ at time $t$, to PG

PGSpikeCount $=$ PGSpikeCount +1

for all neuron $N_{m}$ recieving a connection from $N_{l}$ do

Enqueue an upcoming PSP evoked in $N_{m}$ at time $t+d_{l m}$ by the spike from $N_{l}$

35:

36:

37:

38:

39:

end if

end while

40: $\quad$ if $\quad$ PGSpikeCount $>$ NbSpikeMin then

41: $\quad$ Save the $\mathrm{PG}$

42: $\quad$ end if

43: $\quad$ end if

44: end for

45: end for 